\title{
Amniotic Fluid Embolism: An Update of the Evidence
}

\author{
Isabella Piva, Scutiero G and Greco P \\ Department of Morphology, Surgery and Experimental Medicine, University of Ferrara, Ferrara, Italy
}

Corresponding author: Isabella Piva, Department of Morphology, Surgery and Experimental Medicine, Ferrara University, Corso Giovecca 83, 44121,Ferrara, Italy, Tel: +393497905494; Email: pvisIl@unife.it

Received date: July 19, 2016; Accepted date: August 11, 2016; Published date: August 18, 2016

Citation: Piva I, Scutiero G, Greco P. Amniotic Fluid Embolism: An Update of the Evidence. Med Toxicol Clin Forens Med. $2016,2: 2$.

Copyright: (c) 2016 Piva I, et al. This is an open-access article distributed under the terms of the Creative Commons Attribution License, which permits unrestricted use, distribution, and reproduction in any medium, provided the original author and source are credited.

\section{Abstract}

This article reviews the current evidence on amniotic fluid embolism (AFE), focusing on epidemiology, pathogenesis, clinical presentation, diagnosis and treatment. AFE is a rare and life-threatening obstetric condition, occurring in 2 to 8 per 100000 births. With a mortality of 0.5 to 1.7 deaths per 100,000 deliveries in the developed world and 1.9 to 5.9 deaths per 100,000 deliveries in the developing world, it is among the leading direct causes of maternal death. Furthermore, persistent neurological impairment has been reported in $6-61 \%$ of survivors. Even though there is no shared pathogenetic theory for AFE, the transfer of amniotic fluid components in the maternal circulation may lead either to an inflammatory-mediated anaphylactoid response or to complement activation. There is great variability in the presentation of AFE from classical cardiopulmonary collapse with coagulopathy to minor and subclinical presentations. In severe cases, AFE should be distinguished from other acute and life-threatening emergencies, such as pulmonary embolism, myocardial infarction and peripartal cardiomyopathy. Thus, the diagnosis is one of exclusion, very often on post-mortem report. In fact, several diagnostic biomarkers have been proposed, but they have not been established in routine clinical diagnosis. Prompt recognition of the symptoms and immediate interdisciplinary management are pivotal for prognosis. The traditional treatment is supportive and aimed at treating cardiovascular, pulmonary, and coagulation derangements. Recently, novel treatment strategies have been proposed in the field of invasive hemodynamic support and C1 esterase inhibitors represent a potential therapeutic option. However, due to the lack of specific diagnostic tests, the difficulty of establishing the diagnosis and excluding competing diagnoses, and the complex treatment required, AFE still remains a major clinical challenge.

\section{Keywords:}

Amniotic fluid embolism; Maternal death; Pregnancy; Embolism
How this fits in with quality in primary care?

What do we know?

Amniotic fluid embolism (AFE) is a rare and devastating obstetrical condition characterized by a grim prognosis in terms of survival and morbidity. In the absence of reliable diagnostic markers, the diagnosis is based on the clinical presentation and it is one of exclusion. The traditional treatment is supportive.

What does this paper add?

This paper reviews recent epidemiological data, current evidence about the pathophysiological mechanisms involved in AFE and focuses on novel treatment strategies.

\section{Introduction}

Amniotic fluid embolism (AFE) is a rare and incompletely understood condition that is unique to pregnancy. This disease is characterized by the sudden onset of maternal compromise generally involving the cardio-respiratory and haematological systems, which can rapidly progress to cardiac arrest and profound coagulopathy, leading to death [1]. In this review we aim to extend our previous work, by summarizing the current evidence concerning epidemiology, pathogenesis, diagnosis, and management for AFE [2].

\section{Epidemiology}

Provided the fact that reliable data are difficult to obtain due to heterogeneous diagnostic criteria, the incidence rate is estimated to range from 2 to 8 per 100000 births in different countries [3, 4]. However, a lower incidence (1.7 per 100000 births) has been reported in a prospective population-based study, collecting data of 7001438 maternities in the period between 1 February 2005 and 31 January 2014 in the United Kingdom [5].

AFE is one of the leading causes of death resulting directly from childbirth, as it accounts for $5 \%$ to $15 \%$ of cases worldwide [3]. It is a more frequent cause of death in the developed countries, while it is not dominant in developing ones [6]. The case-related maternal mortality is estimated to range between 0.5 to 1.7 deaths per 100,000 deliveries in the developed world and 1.9 to 5.9 deaths per 100,000 deliveries in the developing 
world [6]. Based on the recent United Kingdom Obstetric Surveillance System (UKOSS) report, maternal mortality rate is even lower and accounts for 0.3 per 100000 maternities5. On the grounds of previous data, the rate of perinatal mortality seemed to range between $7 \%$ and $38 \%$, but it has been recently estimated to be $6.7 \%$ in the UK. This datum is still significantly higher than the national perinatal mortality rate of $0.75 \%[5,7]$.

Between $24 \%$ and $50 \%$ of surviving children suffer from persistent neurological deficits [8]. As compared to AFE being diagnosed before or at delivery, diagnosis of AFE in the postpartum period seems to predispose significantly to perinatal death, need for intensive neonatal care and major complications [5].

\section{Pathogenesis and Pathophysiology}

The pathogenesis of AFE has not been clarified yet. Amniotic fluid (AF) can enter the maternal circulation via endocervical veins, lesions of the uterus, or the site of placental attachment and was once thought to cause a purely mechanical obstruction of the pulmonary vessels [1]. Nowadays there are two current pathogenetic theories for AFE. According to the "anaphylactoid reaction hypothesis", AF contains vasoactive (bradykinin, histamine, and others) and procoagulant substances that can lead to endothelial activation and cause a massive inflammatory reaction [9]. Evidence supporting this hypothesis focuses on the role of $\beta$-tryptase, a serine protease contained into mast cell granules, whose levels rise both in anaphylaxis and in other allergic states. It has been proved that serum tryptase levels are not necessarily above the upper limit in fatal cases of AFE. However, the detection of a significantly higher number of mast cells and significantly higher levels of tryptase at pulmonary level in fatal AFE cases supports mast cell degranulation as the physiopathological mechanism of AFE [9]. On the other hand, the activation of complement could be responsible for the onset of AFE. In fact, complement levels are reported not to undergo a significant change during normal labour, even though they fall immediately after birth [9]. Therefore, abnormally low levels of C3 and C4 detected in AFE cases are suggestive of complement activation, either through the classical or alternative pathway [9, 10]. The susceptibility of women to develop AFE after the transfer of AF or its components may depend on allergic diatheses or previous sensitization to specific fetal antigens [11]. Nevertheless, no related theory based on reliable evidence has been widely accepted nor have amniotic fluid-specific markers been developed, so far.

AFE occurs during labor and delivery/Cesarean section $(55 \%$ to $76 \%$ antenatally) or up to $48 \mathrm{~h}$ postpartum [12]. It may rarely occur during pregnancy following intrauterine surgery (e.g. abortion), blunt abdominal trauma or amniocentesis [3, 13]. Several risk factors may predispose to AFE, including maternal age $\geq 35$ years, placenta previa and multiple pregnancies $[5,12]$. Additionally, instrumental vaginal and caesarean deliveries are associated with the occurrence of AFE post-natally [5]. In fact, the rupture of the membranes followed by the inflow of amniotic fluid into maternal circulation occurs easily in cases of cesarean delivery, forceps/vacuum delivery, placenta previa, abruption placenta and eclampsia, because these conditions are ascribed to injury of the birth canal or injury of trophoblasts [14]. In keeping with this assumption, 91\% women had ruptured membranes at or before AFE presentation in the recent UKOSS survey [5]. According to recent data, even labor induction seems to predispose to AFE $[5,12]$. Interestingly, there is no evidence of a temporal change in the incidence of or risk factors for AFE in recent times [5].

The first phase of AFE develops within 30-60 min after the onset of clinical symptoms. Pulmonary vasoconstriction and acute pulmonary hypertension lead to acute right heart failure with dilatation of the right ventricle and severe tricuspid insufficiency. Altered pulmonary perfusion and damage to the gas exchange surfaces caused by inflammation result in respiratory failure. In the second phase of $A F E$, reactive hypovolemia, cardiodepressive humoral factors from the AF and myo-cardial ischemia may cause acute left heart failure with consequent pulmonary edema (51\% to $100 \%)$. Neurological sequelae and multi-organ failure depend from subsequent hypotension and hypoxia [3]. In $30 \%$ to $45 \%$ of patients coagulopathy develops with severe bleeding resulting from disseminated intravascular coagulation (DIC). Such a phenomenon may develop in two alternative ways: on the one hand, the procoagulant substances contained in the AF may activate the extrinsic coagulation cascade on the other hand, urokinase-like plasminogen activator and plasminogen activator 1 contained in the AF may trigger massive hyperfibrinolysis [9, 11, 15].

\section{Clinical Presentation}

The initial symptoms may be preceded by a non-specific prodromal phase or develop suddenly and the clinical scenario is characterized by different associations of symptoms with variable severity. The main ones include: acute dyspnea (30-40\%) and cyanosis (50\% to $80 \%$ ); sudden hypotension (56\% to $100 \%)$, cardiac arrest $(30 \%$ to $87 \%)$, or fetal distress $(20 \%$ to $36 \%)$ detectable by cardiotocography $[3,5]$. Rarer clinical manifestations are: seizures, acute confusion and, in extreme cases, unconsciousness/coma (15\% to $50 \%)$ or life-threatening hemorrhage resulting from coagulopathy $(\leq 12 \%)[3,16]$. Kanayama and Tamura proposed that two-thirds of AFE cases present with atonic bleeding, and only one-third with cardiopulmonary collapse [14].

According to recent UKOSS data, AFE presented at or before delivery in $53 \%$ of women, at a median gestation of 39 weeks (range 28-42 weeks); 47\% presented with AFE a median of 19 min after delivery (range $1 \mathrm{~min}$ to $6 \mathrm{~h} 27 \mathrm{~min}$ ) having delivered at a median gestation of 39 weeks (range 28-42 weeks) [5].

\section{Diagnosis}

The diagnosis of AFE is based on clinical symptoms. In fact, zinc coproporphyrin, sialyl-Tn antigen, tryptase or C3 and C4 complement and detection of insulin-like growth factor binding protein-1 appear promising diagnostic markers for AFE, but they have not been established in routine clinical diagnosis [3]. Furthermore, hemodynamic parameters, ECG, blood gas analysis, chest X-ray and laboratory tests (including blood count, 
cardiac enzymes, and coagulation tests) and specific tests such as trans-esophageal echocardiography (TEE) and rotational thromboelastometry play a limited role in diagnosis and should be used instead for monitoring and treatment optimization. Thus, the diagnosis of AFE is one of exclusion and should be considered in every case of sudden maternal cardiovascular collapse and/or maternal death in childbirth with unexplained etiology. Although there are no general accepted criteria for diagnosis, reliable parameters were proposed by Benson et al. and later on by the UKOSS study12 (Table 1). According to recent data, at presentation, women had a median of four of the features of AFE and the diagnosis, including both antenatal and postnatal cases, was first considered a median of $33 \mathrm{~min}$ (range 0 min to 2 days) after presentation [5]

Table 1 Criteria for diagnosis of AFE.

\begin{tabular}{|c|c|}
\hline \multirow{2}{*}{$\begin{array}{l}\text { Benson criteria }^{9} \\
\text { Pregnant women up to } 48 \mathrm{~h} \text { after birth with } \\
\text { one or more of the following symptoms } \\
\text { and requiring treatment: }\end{array}$} & UKOSS criteria ${ }^{12}$ \\
\hline & $\begin{array}{l}\text { No other clear cause: acute } \\
\text { cardiovascular collapse with one } \\
\text { or more of the following signs: }\end{array}$ \\
\hline - $\quad$ Hypotension (and/or cardiac arrest) & - $\quad$ Acute fetal compromise \\
\hline - $\quad$ Respiratory distress & Cardiac arrest \\
\hline $\begin{array}{l}\text { - Disseminated intravascular } \\
\text { coagulation }\end{array}$ & Cardiac arrhythmia \\
\hline - $\quad$ Coma and/or seizures & - $\quad$ Coagulopathy \\
\hline \multirow[t]{5}{*}{$\begin{array}{l}\text { No other medical explanation for } \\
\text { clinical course }\end{array}$} & Hypotension \\
\hline & $\begin{array}{l}\text { - Maternal hemorrhage } \\
\text { (Excluding women with maternal } \\
\text { hemorrhage as the first symptom } \\
\text { with no evidence of early } \\
\text { coagulopathy } \\
\text { cardiorespiratory compromise or } \\
\text { in cases of postnatal evidence of } \\
\text { fetal squames or hairs in the } \\
\text { lung) }\end{array}$ \\
\hline & $\begin{array}{l}\text { - Premonitory symptoms, } \\
\text { (e.g. restlessness, anxiety, } \\
\text { agitation) }\end{array}$ \\
\hline & - $\quad$ Seizures \\
\hline & $\begin{array}{l}\text { - (Sudden onset) shortness } \\
\text { of breath }\end{array}$ \\
\hline
\end{tabular}

Differential diagnoses include pulmonary embolism (PTE), myocardial infarction and peripartal cardiomyopathy. The first differs most markedly from AFE in its typical risk factors, chest pain, rarer initial hypotension and usually the absence of coagulopathy. In fact, in PTE, fibrinogen level decreases and Ddimer level rises with increasing pulmonary occlusion rate, but hypofibrinogenemia (defined as $<0.5 \mathrm{~g} / \mathrm{L}$ ) is seldom seen in patients with PTE [17].

Unfortunately, the diagnosis is very often a post-mortem report in case of sudden death during childbirth. In this case, careful histological examination reveals formed AF components such as usually lamellar, adjacent epidermal squames, meconium components, or lanugo hairs in the pulmonary blood flow [12]. AFE can be ruled out if no histological evidence of AF components in the lung is provided in the first three days following clinical manifestation of AFE and maternal death.

\section{Management}

A multidisciplinary team including anaesthesia, respiratory therapy, critical care, and maternal-fetal medicine is recommended. The mainstay of the treatment includes the following procedures:

1) Safeguard the airways: endotracheal intubation and early sufficient oxygenation should be performed using an optimized Fi02: PEEP (positive end-expiratory pressure) ratio. Reliable prevention against aspiration is essential.

2) Hemodynamic balance: crystalloid-based volume replacement and possible early use of vasopressors (e.g. noradrenaline, dobutamine) is recommended [3].

3) Laboratory tests: coagulation tests, cross-matching, blood gas analysis, and -if available-rotational thromboelastometry (to distinguish between hemostatic disorders and assess their severity) should be checked.

4) Central venous catheter or arterial cannula positioning: even though the placement should not delay further treatment.

5) Emergency Caesarian section, with resuscitation facilities, should be performed in 5-7 min in the event of cardiac arrest or life-threatening cardiac arrhythmia. The delivery of the fetus results in a $60-80 \%$ increase in maternal cardiac output and reduces neurological complications for the fetus.

6) Neonatal care should be provided promptly, taken into account the possible neurologic complications in the new-born.

7) Maternal post-partum care is based on several procedures, including: I) prevention of atony through immediate administration of uterotonics and hysterectomy in case of treatment refractarity [7]; II) differentiated use of catecholamines optimized using TEE [16] and possible additional inotropy support; III) cardiac pump function monitoring [16]; IV) prompt optimization of coagulation status through initial administration of tranexamic acid to treat hyperfibrinolysis and subsequent use of fibrinogen concentrate (for fibrinogen levels below $2 \mathrm{~g} / \mathrm{L}$ ) possibly using rotational thromboelastometry [18]; V) Replacement of red blood cell concentrates and fresh-frozen plasma (FFP) according to blood loss/severity of bleeding and in line with the risk profile of the patient, paying attention to volume overload that can possibly lead to pulmonary oedema (Optimal RBC to FFP ratio 1:1-1.5 [14]; VI) administration of recombinant factor VIla if the above treatments are ineffective for improving DIC [14].

In a scenario in which AFE management only includes supportive and palliative measures, novel treatment strategies have also been proposed. Antithrombin concentrates may improve outcomes in patients with AFE that develop coagulopathy [19]. On the contrary, heparin is not recommended because of the high risk of massive hemorrhage in the setting of AFE14. Invasive hemodynamic support may be considered when institutionally available in patients unresponsive to initial resuscitative interventions. 
Extracorporeal membrane oxygenation [20], cardiopulmonary bypass [21], intra-aortic balloon pump [22], pulmonary artery thromboembolectomy [23], hemofiltration [24] and plasma exchange transfusions [25] have been the object of several case reports, but their safety and efficacy needs to be further tested. Plasma exchange may remove chemical mediators and cytokines responsible for the anaphylactoid response [26]. High-dose corticosteroid treatment is also supposed to counteract the inflammatory reaction [21]. Consistent with the complement activation pathogenetic hypothesis, Tamura and coll. reported that mean $\mathrm{C} 1$ esterase inhibitor ( $\mathrm{C} 1 \mathrm{INH})$ activity level in clinical AFE cases was significantly lower than those of normal postpartum women [27]. Therefore, the Authors proposed $\mathrm{C} 1 \mathrm{INH}$ administration as a therapeutic option. In fact, $\mathrm{C} 1 \mathrm{INH}$ is capable not only of inhibiting the complement system but also of modulating the coagulo-fibrinolytic kallikrein-kinin systems. Recently, C1INH was successfully used in a patient presenting uterine atony, shock vitals and bleeding tendency. No anti-DIC agent (fibrinogen, antithrombin or FFP) was applied before the administration of C1INH concentrate; however, the levels of blood fibrinogen and antithrombin showed marginal change and increased, suggesting the independent effect of $\mathrm{C} 1 \mathrm{INH}$ to cease the progression of DIC from AFE [28].

In addition to that, Evans and coll. achieved successful restoration of spontaneous circulation in a case of AFE complicated by pulseless electrical activity, through the use of sodium bicarbonate. On the grounds of their results, the Authors suggested to use sodium bicarbonate in the cases of suspected AFE that are characterized by evident right ventricular failure on TEE and acidosis and/or hypercarbia and do not respond to normal advanced life support measures. This substance is supposed to reduce the pulmonary vascular resistance directly, allowing the failing right ventricle to restore forward flow [29]. The efficacy of sodium bicarbonate had not been proved in previous reports, tough this could be due to the fact that TEE was never used during the initial resuscitation [30].

\section{Prognosis}

More than half of the patients $(56 \%)$ die in the initial phase (0-23 $\mathrm{h}$ after initial clinical manifestations) [12], with death occurring a median of $1 \mathrm{~h}$ and $42 \mathrm{~min}$ after AFE presentation [5]. The leading causes of maternal death in the second phase are sudden cardiac arrest, hemorrhage resulting from coagulopathy or acute respiratory distress syndrome, and/or multiple organ failure [3]. Recently, the prognostic role of cardiac arrest has been emphasized: it seems to be significantly related to fatal outcome and permanent neurological injury, especially in the cases in which it is the first recognized symptom or sign of AFE5. Among survivors, persistent neurological impairment has been reported in $6-61 \%$ of women [8]. As pointed out by Fitzpatrick and coll., fatal outcome and permanent neurological injury are significantly related to cryoprecipitate administration, hysterectomy, shorter time interval between the AFE event and hysterectomy and ethnic provenance (black or other minority ethnic groups) [5].

Early diagnosis and aggressive treatment are the most critical factors associated with survival. The initial goal of the treatment is the rapid correction of maternal hemodynamic instability, ideally operated within an intensive care unit (ICU) by an interdisciplinary team. Accordingly, Fitzpatrick and coll reported that $90 \%$ of women who survived were admitted to an intensive therapy unit/high-dependency unit [5].

\section{Conclusion}

Despite a deeper understanding of its pathophysiology and risk factors, AFE still represents a diagnostic and therapeutic challenge. In the absence of reliable diagnostic markers, AFE should be diagnosed on the grounds of clinical manifestations that are often heterogeneous and unspecific. A prompt identification of the symptoms and a multidisciplinary management play a key role to prevent death and permanent neurological deficits. While supportive procedures constitute the mainstay of clinical management, novel treatment options are promising, but need further evaluation before being included in the therapeutic armamentarium.

\section{Conflict of Interest}

The Authors have no conflict of interest to declare. 


\section{References}

1. Clark SL (2010) Amniotic fluid embolism. Clin Obstet Gynecol 53: 322-328.

2. Pantaleo G, Luigi N, Federica T, Paola S, Margherita N, et al. (2014) Amniotic fluid embolism: Review. Curr Pharm Biotechnol 14: 1163-1167.

3. Conde-Agudelo A, Romero R (2009) Amniotic fluid embolism: An evidence-based review. Am J Obstet Gynecol 201: 445.

4. Kramer MS, Rouleau J, Liu S, Bartholomew S, Joseph KS (2012) Maternal Health Study Group of the Canadian Perinatal Surveillance System. Amniotic fluid embolism: Incidence, risk factors and impact on perinatal outcome. BJOG 119: 874-879.

5. Fitzpatrick KE, Tuffnell D, Kurinczuk JJ, Knight M (2016) Incidence, risk factors, management and outcomes of amniotic-fluid embolism: A population-based cohort and nested case-control study. BJOG 123: 100-109.

6. Mo X, Feng A, Liu X, Tobe RG (2014) Amniotic fluid embolism (AFE) in China: Are maternal mortality and morbidity preventable? Intractable Rare Dis Res 3: 97-99.

7. Tuffnell D, Knight M, Plaat F (2011) Amniotic fluid embolism - an update. Anaesthesia 66: 3-6.

8. Tuffnell DJ (2005) United kingdom amniotic fluid embolism register. BJOG 112: 1625-1629.

9. Benson MD (2007) A hypothesis regarding complement activation and amniotic fluid embolism. Med Hypotheses 68: 1019-1025.

10. Hammerschmidt DE, Ogburn PL, Williams JE (1984) Amniotic fluid activates complement. A role in amniotic fluid embolism syndrome? J Lab Clin Med 104: 901-907.

11. Gist RS, Stafford IP, Leibowitz AB, Beilin Y (2009) Amniotic fluid embolism. Anesth Analg 108: 1599-1602.

12. Knight $M$, Tuffnell $D$, Brocklehurst $P$, Spark $P$, Kurinczuk JJ; UK Obstetric Surveillance System (2010) Incidence and risk factors for amniotic-fluid embolism. Obstet Gynecol 115: 910-917.

13. Drukker L, Sela HY, loscovich A, Samueloff A, Grisaru-Granovsky S (2016) Amniotic fluid embolism: A rare complication of secondtrimester amniocentesis. Fetal Diagn Ther.

14. Kanayama N, Tamura N (2014) Amniotic fluid embolism: Pathophysiology and new strategies for management. J Obstet Gynaecol Res 40: 1507-1517.

15. Estellés A, Gilabert J, Andrés C, España F, Aznar J (1990) Plasminogen activator inhibitors type 1 and type 2 and plasminogen activators in amniotic fluid during pregnancy. Thromb Haemost 64: 281-285.

16. Dean LS, Rogers RP 3rd, Harley RA, Hood DD (2012) Case scenario: Amniotic fluid embolism. Anesthesiology 116: 186-192.

17. Kucher N, Kohler HP, Dornhofer T, Wallmann D, Lammle B (2002) Accuracy of $\mathrm{D}$-dimer/fibrinogen ratio to predict pulmonary embolism: A prospective diagnostic study. J Thromb Haemost 1:708-713.

18. Collins NF, Bloor M, Mc Donnell NJ (2013) Hyperfibrinolysis diagnosed by rotational thromboelastometry in a case of suspected amniotic fluid embolism. Int J Obstet Anest 2013; 22: 71-76.

19. Kobayashi H (2015) Amniotic fluid embolism: Anaphylactic reactions with idiosyncratic adverse response. Obstet Gynecol Surv 70: 511-517.

20. Sharma NS, Wille KM, Bellot SC, Diaz-Guzman E (2015) Modern use of extracorporeal life support in pregnancy and postpartum. ASAIO J 61: 110-114.

21. Stanten RD, Iverson LI, Daugharty TM, Lovett SM, Terry C, et al. (2003) Amniotic fluid embolism causing catastrophic pulmonary vasoconstriction: Diagnosis by transesophageal echocardiogram and treatment by cardiopulmonary bypass. Obstet Gynecol 102: 496-498.

22. Hsieh YY, Chang CC, Li PC, Tsai HD, Tsai CH (2000) Successful application of extracorporeal membrane oxygenation and intraaortic balloon counterpulsation as lifesaving therapy for a patient with amniotic fluid embolism. Am J Obstet Gynecol 183: 496-497.

23. Esposito RA, Grossi EA, Coppa G (1990) Successful treatment of postpartum shock caused by amniotic fluid embolism with cardiopulmonary bypass and pulmonary artery thromboembolectomy. Am J Obstet Gynecol 163: 572-574.

24. Weksler N, Ovadia L, Stav A, Ribac L, luchtman M (1994) Continuous arteriovenous hemofiltration in the treatment of amniotic fluid embolism. Int J Obstet Anesth 3: 92-96.

25. Dodgson J, Martin J, Boswell J, Goodall HB, Smith R (1987) Probable amniotic fluid embolism precipitated by amniocentesis and treated by exchange transfusion. Br Med J (Clin Res Ed) 294: 1322-1323.

26. Ogihara T, Morimoto K, Kaneko Y (2012) Continuous hemodiafiltration for potential amniotic fluid embolism: dramatic responses observed during a 10 year period report of three cases. Ther Apher Dial 2012; 16:195-197.

27. Tamura N, Kimura S, Farhana M, Uchida T, Suzuki K, et al. (2014) C1 esterase inhibitor activity in amniotic fluid embolism. Crit Care Med 42: 1392-1396.

28. Todo Y, Tamura N, Itoh H, Ikeda T, Kanayama N (2015) Therapeutic application of C1 esterase inhibitor concentrate for clinical amniotic fluid embolism: A case report. Clin Case Rep 3: 673-675.

29. Evans S, Brown B, Mathieson M, Tay S (2014) Survival after an amniotic fluid embolism following the use of sodium bicarbonate. BMJ Case Rep.

30. Mahshid N, Ahmad S, Nahid M, Afshin F (2009) Sudden cardiac arrest during cesarean section - A possible case of amniotic fluid embolism. Middle East J Anaesthesiol 20: 315-317. 